Results Between 1977 and 2003, all types of road traffic crashes increased markedly in Sri Lanka: total (194.8\%), fatal $(81.8 \%)$, grievous $(257.9 \%)$, non-grievous $(69.2 \%)$ and damage only $(303.5 \%)$. However a reduction was observed in damage only (69.1), non-grievous (16.8\%) and total (48.2\%) crashes from 2003 to 2007 . However, fatal crashes and grievous crashes increased by $11.0 \%$ and $24.5 \%$ respectively.

Conclusion Since 2003, damage only, and non-grievous crashes decreased markedly. However such a reduction was not seen in more serious fatal and grievous crashes. In 2003, Sri Lankan insurance companies changed their insurance policies and introduced an on-the-spot payment scheme. No law amendment was introduced in 2003. Therefore the reduction of damage only, and non-grievous crashes could be due to the high under reporting of crashes, rather than an actual reduction.

\title{
0921 TRENDS OF ROAD TRAFFIC CRASHES IN SRI LANKA
}

A U Jayatilleke*, A C Jayatilleke Correspondence: Department of Community and Global Health, Graduate School of Medicine, University of Tokyo, Hongo 7-3-1, Bunkyo ku, Tokyo 113-0033, Japan

10.1136/ip.2010.029215.921

Introduction Sri Lanka is a lower middle-income country in the South Asian Region which is facing a growing burden of road traffic crashes (RTCs). In Sri Lanka, RTCs lead to more than 2000 deaths and 14000 casualties every year. We conducted this study to examine the trends of RTCs in Sri Lanka for the 30 year between 1977 and 2007.

Methods We extracted the police reported RTCs between 1977 and 2007 from the statistics unit, police traffic headquarters, Sri Lanka. We calculated the crash and the death rates per 100000 population and analysed the trends of RTCs during the 30 years. We plotted graphs to show the trend or the RTCs during this period. 